\title{
Nonlinear Boundary Value Problems and Orlicz Spaces $\left(^{*}\right)$.
}

R. Kannay (St. Louis, Mo., U.S.A.) - J. D. SchuUR (East Lansing, Mich., U.S.A.)

Sunto. - Usando metodi dell'analisi funzionale si ottengono teoremi di esistenza delle soluzioni di un'equazione della forma $L x+N x=0$ dove $L \dot{e}$ un operatore differenziale ordinario lineare autoaggiunto di ordine pari, positivo, mentre $N$ è un operatore non necessariamente lineare.

\section{1. - Introduction.}

In this paper we use functional analytic techniques to show the existence of solutions to boundary value problems of the form

$$
L x+N x=0
$$

where $L$ is an even order, positive, self-adjoint, linear ordinary differential operator and $N$ is an operator, usually nonlinear.

The following theorem is an example of the type of problem we first considered.

Theorem 1.1. - Let $f(t, x, y)$ be continuous from $D=\{(t, x, y): 0 \leq t \leq 1,|x| \leq R$, $|y|<\infty\}$ into $R^{n}$ and let $f$ satisfy

a) $x \cdot f(t, x, y)+|y|^{2} \geqslant 0$ when $x \cdot y=0$ and $|x|=R$;

b) $|f(t, x, y)| \leq \varphi(|y|)$ where $\varphi:[0, \infty) \rightarrow(0, \infty)$ is continuous and $\int^{\infty} r d r / \varphi(r)=\infty$ (a Nagumo condition);

c) $|f(t, x, y)| \leq 2 K\left(x \cdot f(t, x, y)+|y|^{2}\right)+L, K$ and $L$ non-negative constants.

Then the problem

$$
x^{\prime \prime}=f\left(t, x, x^{\prime}\right), \quad x(0)=x(1), \quad x^{\prime}(0)=x^{\prime}(1)
$$

has at least one solution in $D$.

Here $x$ and $y$ are $n$-vectors, $x \cdot y$ is the dot product, and $|x|=(x \cdot x)^{\frac{1}{2}}$.

For the two point boundary value problem a theorem of this type was proved by NAGUMo [16] and, for the vector case, by HARTMAn [6]. The development of Theorem 1.1 may be followed in [6], [11], [17] and [2]. A resume is given in [7, Chap. XII].

(*) Entrata in Redazione il 4 giugno 1976 . 
With $L x=-x^{\prime \prime}$, and the boundary conditions incorporated into the domain of $L$, and with $N$ the nonlinear Nemytskii operator generated by $f,(1.2)$ can be written in the form (1.1).

Since the null space of $L$ is non-trivial we choose to use the "method of alternative problems " (see CESARI [3], HALE [8], or MAWHIN [15]) to attack the problem. We can then give an operator-theoretic proof for a class of problems like (1.2) with regard to which the following are of interest:

1) Certain higher order operators $L$, with a finite dimensional null space, can be considered.

2) Condition (a) of Theorem 1.1 becomes a condition on vector fields to be used with Leray-Schauder degree theory.

3) The problem may be put into a natural Orlicz space setting and $(b)$ of Theorem 1.1 is replaced by a growth condition on the $N$-function determining the space. For example, we can allow $|f(t, x, y)| \leq|y|^{p}, p>1$ an integer, and prove our theorem.

Contents of the paper. Section 2: A list of notations and function spaces used in the paper. Section 3: An operator-theoretic proof of a version of Theorem 1.1. Section 4: An abstract version of the theorem.

\section{2. - Notation and spaces.}

We list here the several function spaces and some notation we use in subsequent sections.

a) Euclidean $n$-space will be denoted by $R^{n}$, the norm will be $|x|=(x \cdot x)^{\frac{1}{t}}$ where $x \cdot y=\sum_{1}^{n} x_{i} y_{i}$.

b) The space of continuous functions from $[0,1]$ into $R^{n}$ with norm $\|x(\cdot)\|_{0}=$ $=\sup \{|x(t)|: 0 \leq t \leq 1\}$ will be denoted by $C[0,1]$. The subset of absolutely continuous functions will be denoted by $A C[0,1]$; the subset of functions with $n$ continuous derivatives, by $C^{n}[0,1]$; and the subset of functions with derivatives of every order, by $O^{\infty}[0,1]$.

c) The Lebesgue spaces, with the usual norm, will be denoted by $L_{p}[0,1]$ and $\|\cdot\|_{p}$, respectively, where $p \geqslant 1$. Often we shall write $L_{p}$ for $L_{p}[0,1]$ and $\|\cdot\|$ for $\|\cdot\|_{2}$. The inner product on $L_{2}$ will be denoted by $(\cdot, \cdot)$.

d) For Orlicz spaces we shall assume an acquaintance with KRASNOSEL'SKII and RutTckin [13]. (Or, see [5].) To facilitate reading one can think of $\Phi(u)=u^{p}$ and $L_{\phi}=L_{p}, p>1$. Our theorems do allow functions that grow faster than $u^{p}$. 
i) Let $\Phi(u)$ and $\Psi(u)$ be complementary $N$-functions and let $L_{\Psi}=L_{\Phi}[0,1]$, with norm $\|\cdot\|_{\Phi}$, and $L_{\Psi}=L_{\Psi}[0,1]$, with norm $\|\cdot\|_{\Psi}$, be the corresponding Orlicz spaces $[13$, pp. 11, 67].

ii) If $\Psi(u)$ satisfies a $\Delta_{2}$-condition, then the bounded, measurable functions, and hence the $L_{2}$-functions, are dense in $L_{\Psi} ; L_{\Psi}$ is separable; and $L_{\Psi}^{*}=L_{\Phi}$ (where * denotes the dual space) $[13, \mathrm{pp} .81,130]$.

iii) If $\Psi(u)<u^{2}<\Phi(u)$, then $L_{\Phi} \subset L_{2} \subset L_{\Psi}$ and $\|\cdot\| \psi \leq k \|_{\Phi}$ for some constant $k>0\left[13\right.$, pp. 110, 112]. And if $u^{2}<\Phi(u)$, then $\Psi(u)$, its complementary $N$-function, satisfies $\Psi(u)<u^{2}$ (see $[13$, p. 16]).

iv) Hence if $\Psi(u)<u^{2}<\Phi(u)$ and $\Psi(u)$ satisfies a $\Delta_{2}$-condition we can say: If $x \in L_{\Phi}=L_{\psi}^{*}, y \in\left(L_{\Psi} \cap L_{2}\right)$, and $\langle x, y\rangle$ denotes the usual pairing between an element $y$ in a space $X$ and $x$ in $X^{*}$, then $\langle x, y\rangle=(x, y)$ (the $L_{2}$-inner product). When this conclusion and the conclusions of (ii) and (iii) hold, we say that $\left(L_{\Psi}, L_{2}, L_{\Phi}\right)$ are in normal position (see [1]).

v) If $\Phi(u)$ satisfies a $\Delta_{3}$-condition, then $\Psi(u)$ satisfies a $\Delta_{2}$-condition. The functions $\Phi(u)=u^{y}$ or $\Phi(u)=\exp \left(u^{p}\right), p>1$, satisfy $\Delta_{3}$-conditions [13, p. 35].

e) The Sobolev space $\left\{x(t): x, x^{\prime}, \ldots, x^{(n-1)} \in A O[0,1], x^{(n)} \in L_{2}\right\}$ with norm $\|\cdot\|_{H}=\|x\|_{0}+\ldots+\pi x^{(n-1)}\left\|_{0}+\right\| x^{(n)} \|$ will be denoted by $H^{n}[0,1]$. With a different norm we could have $H^{n}[0,1]$ a Hilbert space. But this norm makes one step in a later proof easier. For a diseussion of this space see [14].

f) The Orlicz-Sobolev space $\left\{x(t): x, \ldots, x^{(n-1)} \in A C[0,1], x^{(n)} \in L_{\phi}\right\}$ with norm $\|\cdot\|_{H \Phi}=\|x\|_{0}+\ldots+\left\|x^{(n-1)}\right\|_{0}+\left\|x^{(n)}\right\|_{\infty}$ will be denoted by $\boldsymbol{H}_{\Phi}^{n}[0,1]$. For a discussion of this space see [5].

g) If $A$ is an operator the domain, range, and null space $A$ will be denoted by $D_{A}, R_{A}$, and $N_{A}$, respectively.

\section{3. - A second order problem.}

THEOREM 3.1. - Let $f(t, x, y):[0,1] \times R^{n} \times R^{n} \rightarrow R^{n}$ be continuous and satisfy:

a) $|f(t, x, y)| \leq a+b|x|+c|y|^{p}$ for some positive constants $a, b$, $c$ and some integer $p>1$; and

b) $\left\|x^{\prime}(\cdot)\right\|^{2}+\int_{0}^{1}\left\{x(t) \cdot f\left(t, x(t), x^{\prime}(t)\right)\right\} d t \geqslant 0$ on $\partial B_{r}$ where $B_{r}=\left\{x \in H_{\phi}[0,1]:\|x\|_{0}^{2}+\right.$ $\left.+\left\|a^{\prime}\right\|_{\Phi}^{2} \leq r^{2}\right\}$ for some $r>0$. Here $H_{\Phi}$ is the Orlicz-Sobolev space with $N$-function $\Phi(s)=\delta^{p}$. (See Sections 2.f and 2.e for this terminology.)

Then the boundary value problem

$$
x^{\prime \prime}=f\left(t, x, x^{\prime}\right), \quad x(0)=x(1), \quad x^{\prime}(0)=x^{\prime}(1)
$$

has at least one solution in $B_{r} \cap C^{2}[0,1]$. 
Remark. - Other $N$-functions are admissible-see Theorem 4.1.

Proof. - Let $L$ be the positive, self-adjoint, linear differential operator defined by $D_{L}=\left\{x \in H^{2}[0,1]: x(0)=x(1), x^{\prime}(0)=x^{\prime}(1)\right\}, L x=-x^{\prime \prime}$ and let $N$ be the nonlinear Nemytskii operator defined by $N x=f\left(\cdot, x(\cdot), x^{\prime}(\cdot)\right)$ where $D_{N}$ will be selected later in such a way that $R_{N}$ will lie in the domains of operators applied to $N$.

Then (3.1) can be rewritten as

$$
L x+N x=0
$$

where a solution is in $D_{L} \cap D_{H} \cap C^{2}[0,1]$.

Define the projection operator $P$ by

$$
P x=\int_{0}^{1} x(t) d t, \quad D_{P}=L_{1}[0,1] .
$$

If we let $P\left(L_{1}\right)=T_{0}$ and $(I-P)\left(L_{1}\right)=T_{1}$, then $L_{1}[0,1]=T_{0} \oplus T_{1}$. We shall often restrict $P$ to subspaces of $L_{1}[0,1]$, e.g., $L_{2}$ or $H^{2}$, without comment and we note that $T_{0}$ is the constant functions and $N_{L}=T_{0}$.

Also, if $L_{2}[0,1]=S$ and $\left(S \cap T_{i}\right)=S_{i}(i=1,2)$, then $S=S_{1} \oplus S_{0}$ and in this case we have $S_{1} \perp S_{0}$.

Define the operator $\tilde{H}$ by

$$
D_{\tilde{H}}=s_{1}, \quad \tilde{H} x=\int_{0}^{1}(1-t) s x(s) d s+\int_{i}^{1} t(1-s) x(s) d s+x(0) .
$$

If $H=\tilde{H} \mid R_{L}$, then

$$
H=\left(L \mid\left(D_{L} \cap S_{1}\right)\right)^{-1} \quad \text { and } \quad R_{H}=\left(D_{L} \cap S_{1}\right) .
$$

If we only require that a solution of (3.2) be in $D_{L} \cap D_{N}$, then following the method of alternative problems, see [4], (3.2) is equivalent to

$$
\begin{gathered}
x+H(I-P) N x=x_{0}, \quad x_{0} \in \mathcal{S}_{0}, x \in \mathcal{S} \\
P N x=0 .
\end{gathered}
$$

But a solution $x$ to $(3.5),(3.6)$ will be in $D_{L} \subset H^{2}$. Then $N x \in C[0,1]$ and hence $H(T-P) N x \in C^{2}[0,1]$ and $x \in C^{2}[0,1]$, so $x$ is a true solution of (3.1).

We now rewrite (3.5), (3.6) by using a natural decomposition of $H(I-P)$ into the form $J^{*} J$. This technique has been studied in detail by KANNAN and Locker [9]; here we shall simply outline the method.

Define the operators $T$ and $T^{*}$ by

$$
D_{T}=\left\{x \in H^{1}[0,1]: x(0)=x(1)\right\}, \quad T x=x^{\prime} \text { and } T^{*}=-T .
$$

The operator $L$ equals $T T^{*} ; T^{*}$ is the adjoint of $T$; and $N_{T}=\left(D_{T} \cap S_{0}\right)=N_{T^{*}}$. 
Let $J=\left[T \mid D_{T} \cap S_{1}\right]^{-1}$ and $J^{*}=\left[T^{*}\left[D_{L} \cap S_{1}\right]^{-1}\right.$. Then $J: R_{L} \rightarrow\left(D_{T} \cap S_{1}\right)$, $J^{*}:\left(D_{T} \cap S_{1}\right) \rightarrow\left(D_{L} \cap S_{1}\right)$, and $H=J^{*} J$. And we can represent $J$ and $J^{*}$ as

$$
J x=\int_{0}^{t} x(s) d s-P\left[\int_{0}^{t} x(s) d s\right]=-J^{*} x .
$$

With $x-x_{0}=x_{1},(3.5),(3.6)$ can be rewritten as

$$
\begin{gathered}
x_{1}+J^{*} J(I-P) N\left(x_{1}+x_{0}\right)=0 \\
P N\left(x_{1}+\infty_{0}\right)=0 .
\end{gathered}
$$

With $x_{1}=J^{*} y_{1}, y_{1} \in S_{1}$ and using that $J$ is one-to-one, the above system is equivalent to

$$
\begin{gathered}
y_{1}+J(I-P) N\left(J^{*} y_{1}+x_{0}\right)=0 \\
P N\left(J^{*} y_{1}+x_{0}\right)=0
\end{gathered} \quad y_{1} \in S_{1}, x_{0} \in S_{0} .
$$

The first part of the plan for solving (3.8), (3.9) is based on hypothesis $(a)$ which tells us that if $x \in H_{\Phi}^{1}[0,1]$, with $\Phi(s)=s^{p}$, then

$$
\int_{0}^{1}\left|f\left(t, x(t), x^{\prime}(t)\right) d t\right| \leq M+\int_{0}^{1}\left|x^{\prime}(t)\right|^{p} d t<\infty,
$$

i.e., $N x \in L_{1}$. (See Section 2 for the definition of spaces.)

We shall restrict $J^{*}$ to $\left(L_{\Phi} \cap S_{1}\right)$. Then

$$
\left(L_{\Phi} \cap S_{1}\right) \stackrel{J^{*}}{\longrightarrow} H_{\Phi}^{1} \stackrel{(I-P) N}{\longrightarrow}\left(L_{1} \cap T_{1}\right) \stackrel{y}{\longrightarrow}\left(A C \cap S_{1}\right) \subset\left(L_{\Phi} \cap S_{1}\right)
$$

And we shall show that $J(I-P) N J^{*}$ is compact. But we need an adjustment since $L_{1} \nsubseteq D_{J}$.

Let $K^{*}$ have the same representation as $J^{*}$ in (3.7) and let $D_{K^{*}}=L_{\Phi} \cap S_{1}$; let $K$ have the same representation as $J$ in (3.7) and let $D_{K}=L_{1} \cap T_{1}$. If $x \in D_{K}$, then $K x \in S_{1} \cap A C[0,1]$.

We shall solve the problem

$$
\begin{gathered}
z_{1}+K(I-P) N\left(K^{*} z_{1}+x_{0}\right)=0, \quad z_{1} \in L_{\Phi} \cap S_{1}, x_{0} \in S_{0} \\
P N\left(K^{*} z_{1}+x_{0}\right)=0 .
\end{gathered}
$$

The solution $z_{1}$ (we need not worry about the constant functions in the discussion of this point) will be in $L_{\Phi} \cap S_{1}$. Then $N\left(K^{*} z_{1}+x_{0}\right)$ will be in $L_{1}$ and indeed $z_{1}$ will be absolutely continuous. Repeating this procedure we will have $z_{1}$ 
in $C^{1}$ and thus $z_{1}$ is in the domain of $J^{*},(I-P) N\left(J^{*} z_{1}+x_{0}\right)$ is in the domain of $J$, and $z_{1}$ is a solution of (3.8), (3.9).

To show: $K(I-P) N K^{*}: L_{\Phi} \rightarrow L_{\Phi}$ is continuous and compact.

To show that $K: L_{\Phi} \rightarrow H_{\Phi}^{1} \cap S_{1}$ is continuous and bounded is straightforward; we shall show that $N: H_{\Phi}^{1} \rightarrow L_{1}$ is continuous and bounded; $(I-P)$ is a projection; and to show that $K: L_{1} \rightarrow L_{\Phi}$ is continuous and compact is again straightforward.

$N: H_{\Phi}^{1} \rightarrow L_{1}$ is bounded:

$$
\|N x\|_{1} \leq a+b \int_{0}^{1}|x(t)| d t+c \int_{0}^{1}\left|x^{\prime}(t)\right|^{p} d t \leq a+d\|x\|_{H \varphi} .
$$

$N: H_{\Phi}^{1} \rightarrow L_{1}$ is continuous:

$$
\begin{aligned}
\|N x-N y\|_{1} & \leqq \int_{0}^{1}\left|f\left(t, x(t), x^{\prime}(t)\right)-f\left(t, y(t), x^{\prime}(t)\right)\right| d t \\
& +\int_{0}^{1}\left|f\left(t, y(t), x^{\prime}(t)\right)-f\left(t, y(t), y^{\prime}(t)\right)\right| d t .
\end{aligned}
$$

The first integral: With $x^{\prime}(t) \in L_{p}$,

$$
g(t, c)=f\left(t, c, x^{r}(t)\right)
$$

is continuous in $e$ and measurable in $t$. Hence by Kransnosel'skii's version of Lusin's theorem [12] we may divide $[0,1]$ into disjoint subsets $I_{1}$ and $I_{2}$ such that

$I_{1}$ is closed and $g \mid I_{1} \times R^{n}$ is continuous;

$I_{2}$ has arbitrarily small measure.

We have just seen that the integrands are bounded so we can choose $I_{2}$ such that the integral over $I_{2}$ is less than $\varepsilon / 4$. On $I_{1} \times\left\{x:|x-y(t)|_{0} \leq 1\right\}, f$ is uniformly continuous, hence if $|x(t)-y(t)|_{0}$ is sufficiently small, the integral over $I_{1}$ is less than $\varepsilon / 4$.

The second integral is handled in a similar manner.

Now we use hypothesis $(b)$ and degree theory to solve (3.10), (3.11).

Let $L_{\Phi}=R_{1} \oplus R_{0}$ where $R_{i}=L_{\Phi} \cap T_{i}(i=1,2)$, see (3.3), and define $T: R_{1} \oplus$ $\oplus R_{0} \rightarrow R_{1} \oplus R_{0}$ by

$$
T\left(\begin{array}{l}
z_{1} \\
x_{0}
\end{array}\right)=\left(\begin{array}{c}
K(I-P) N\left(K^{*} z_{1}, x_{0}\right) \\
-x_{0}+P N\left(K^{*} z_{1}, x_{0}\right)
\end{array}\right), \quad N\left(K^{*} z_{1}, x_{0}\right)=N\left(K^{*} z_{1}+x_{0}\right)
$$

Then $T$ is continuous and compact and $(3.10),(3.11)$ is equivalent to

$$
(I+T)\left(z_{1}, x_{0}\right)=0 .
$$


Let $L_{\Phi}^{*}=R_{1}^{*} \oplus R_{0}^{*}$ where $R_{i}^{*}=L_{\Phi}^{*} \cap T_{i}(i=1,2)$. If $w=z_{1}+x_{0} \in L_{\Phi}, \tilde{w}=\tilde{z}_{1}+$ $+\tilde{x}_{0} \in L_{\Phi}^{*}$, the usual pairing $\langle\tilde{w}, w\rangle=\left\langle\tilde{z}_{1}, z_{1}\right\rangle+\left\langle\tilde{x}_{0}, x_{0}\right\rangle$ since

$$
\left\langle z_{1}, x_{0}\right\rangle=\int_{0}^{1}\{[\tilde{w}(t)-P \tilde{w}][P w]\} d t=[P(I-P) \tilde{w}][P w]=0
$$

and likewise $\left\langle\tilde{x}_{0}, z_{1}\right\rangle=0$.

Since $L_{\Phi} \subset L_{\Phi}^{*}$ we can form the pairing $\left\langle(I+\lambda T)\left(z_{1}, x_{0}\right),\left(z_{1}, x_{0}\right)\right\rangle$ for $0<\lambda<1$ and this is greater than $\lambda\left\langle(I+T)\left(z_{1}, x_{0}\right),\left(z_{1}, x_{0}\right)\right\rangle$ if $\left.\left\langle z_{1}, z_{1}\right\rangle+\left\langle x_{0}, x_{0}\right\rangle=r^{2}\right\rangle 0$. Thus if $\left\langle(I+T)\left(z_{1}, x_{0}\right),\left(z_{1}, x_{0}\right)\right\rangle \geqslant 0$ on $\left.\partial B_{r}=\left\{\left(z_{1}, x_{0}\right):\left\langle z_{1}, z_{1}\right\rangle+\left\langle x_{0}, x_{0}\right\rangle=r^{2}\right\rangle 0\right\}$, then $\operatorname{deg}\left(I+T, B_{r}, 0\right)=\operatorname{deg}\left(I, B_{r}, 0\right)=1$ and (3.12) has a solution in $\left(B_{r} \cup \partial B_{r}\right)$. And

$$
\begin{aligned}
\left\langle( I + T ) \left( z_{1},\right.\right. & \left.\left.x_{0}\right),\left(z_{1}, x_{0}\right)\right\rangle=\left\langle z_{1}, z_{1}\right\rangle+\left\langle x_{0}, x_{0}\right\rangle \\
& +\left\langle K(I-P) N\left(K^{*} z_{1}, x_{0}\right), z_{1}\right\rangle+\left\langle-x_{0}+P N\left(K^{*} z_{1}, x_{0}\right), x_{0}\right\rangle \text { (see (i) below) } \\
& =\left\langle z_{1}, z_{1}\right\rangle+\left\langle(I-P) N\left(J^{*} z_{1}, x_{0}\right), J^{*} z_{1}\right\rangle+\left\langle P N\left(K^{*} z_{1}, x_{0}\right), x_{0}\right\rangle \\
& =\left\langle z_{1}, z_{1}\right\rangle+\left\langle N\left(J^{*} z_{1}, x_{0}\right), J^{*} z_{1}+x_{0}\right\rangle-\left\langle P N\left(K^{*} z_{1}, x_{0}\right), J^{*} z_{1}\right\rangle \\
& -\left\langle(I-P) N\left(K^{*} z_{1}, x_{0}\right), x_{0}\right\rangle=\left(\text { see (ii), below) } \int_{0}^{1}\left|x^{\prime}(t)\right|^{2} d t\right. \\
& +\int_{0}^{1}\left\{x(t) \cdot f\left(t, x(t), x^{\prime}(t)\right)\right\} d t \geqslant 0
\end{aligned}
$$

on $B_{r}$ by hypothesis $(b)$. So we are done.

i) Here $K:\left(L_{1} \cap S_{1}\right) \rightarrow\left(L_{\Phi} \cap S_{1}\right)$ and $z_{1} \in L_{\Phi}$. So the dual operator $K^{\prime}:\left(L_{\Phi} \cap S_{1}\right) \rightarrow L^{\infty}$. But on $\left(L_{\Phi} \cap S_{1}\right) \subset\left(L_{2} \cap S_{1}\right), K^{\prime}=J^{*}$. The second pairing here is between an element in $L_{1}$ and one in $L^{\infty}$.

ii) With $J^{*} \varkappa_{1}+x_{0}=x, z_{1}=x^{\prime}$. The last two pairings vanish by computation since again the integral of the pairing acts like $P$.

\section{4. - A general theorem.}

In this section we will extend Theorem 3.1 by considering higher order, more general operators $L$ and more general boundary conditions. To simplify notation we shall assume that all functions are real valued.

Let $\tau x(t)=\sum_{i=0}^{n} a_{i}(t) x^{(i)}(t)$ be a formal differential operator with coefficients $a_{i}(t) \in$ $\in C^{\infty}[0,1]$ and $a_{n}(t) \neq 0$ on $[0,1]$, and let

$$
B_{i}(x)=\sum_{j=0}^{n-1}\left[a_{i j} x^{(j)}(0)+\beta_{i j} x^{(j)}(1)\right], \quad i=1, \ldots, k
$$


be a set of $k$ linearly independent boundary values with the $\alpha_{i j}, \beta_{i j}$ being real constants and $0 \leq k \leq 2 n$. Let $\tau^{*}$ be the formal adjoint of $\tau$ and let $B_{i}^{*}(x), 1 \leq i \leq$ $\leq 2 n-k$, be a set of linearly independent, adjoint boundary values. Define the operators $T, T^{*}$, and $L$ by

$$
\begin{array}{ll}
D_{T}=\left\{x \in H^{n}[0,1]: B_{i}(x)=0, i=1, \ldots k\right\}, & T x=\tau x ; \\
D_{T^{*}}=\left\{x \in H^{n}[0,1]: B_{i}^{*}(x)=0, i=1, \ldots, 2 n-k\right\}, & T^{*} x=\tau^{*} x ;
\end{array}
$$

and

$$
\begin{array}{r}
D_{L}=\left\{x \in H^{2 n}[0,1]: B_{i}^{*}(x)=0=B_{j}\left(\tau^{*} x\right), i=1, \ldots, 2 k-1, j=1, \ldots, k\right\} ; \\
L x=\tau \tau^{*} x .
\end{array}
$$

The following hold:

A) If $\alpha_{i j}=0=\beta_{i j}(1 \leq i \leq k, 0 \leq j \leq n-1)$, then $T$ is a closed, densely-defined linear operator in $S=L_{2}[0,1]$ with $\operatorname{dim} N_{T}=n, R_{T}=S$. Further, $T \mid\left(D_{T} \cap N_{T}^{\frac{1}{T}}\right)$ is a closed, $1-1$ operator and $\left[T \mid\left(D_{T} \cap N_{T}^{\frac{1}{T}}\right)[-1\right.$ is a $1-1$ completely continuous operator, in fact an integral operator, defined on all of $S$. Similar statements can be made with respect to $T^{*}$ and, $L$. For a reference see Locker [14].

B) $L$ admits a decomposition, $L=T T^{*}$, where $T^{*}$ is the adjoint of $T$; $N_{T^{*}}=N_{L}\left(=N_{T}\right), R_{T}=R_{L}$, and $L_{2}=S=S_{1} \oplus S_{0}$ where $S_{1}=R_{L}, S_{0}=N_{L}$;

$$
T^{*}:\left(D_{L} \cap B_{1}\right) \rightarrow\left(D_{T} \cap B_{1}\right), \quad T:\left(D_{T} \cap B_{1}\right) \rightarrow R_{L}
$$

and thus restricted these mappings are 1-1 and onto; and the inverses of $T$ and $T^{*}$, which we shall denote by $J$ and $J^{*}$, respectively, exist, are completely continuous, and in fact are integral operators.

THeoremi 4.1. - Let $f\left(t, x_{0}, \ldots, x_{n}\right)$ be continuous on $[0,1] \times R \times \ldots \times R$ and let $N x=f\left(\cdot, x(\cdot), \ldots, x^{(n)}(\cdot)\right)$. If $\left.a\right)\left|f\left(t, x_{0}, \ldots, x_{n}\right)\right| \leq a+b_{0}\left|x_{0}\right|+\ldots+b_{n}\left|x_{n-1}\right|+\Phi\left(\left|x_{n}\right|\right)$, where $\Phi(u)$ is an $N$-function with complementary $N$-function $\Psi(u)$ such that $\Psi<u^{2}<\Phi(u)$ and $\Psi(u)$ satisfies a $\Delta_{2}$-condition (see Section $2 . d$ ); and if

b) $\left\|x^{(n)}(\cdot)\right\|^{2}+\int_{0}^{1}\left\{x(t) f\left(t, x(t), \ldots, x^{(n)}(t)\right)\right\} d t \geqslant 0$ on $\partial B_{r}$, where

$$
B_{\gamma}=\left\{x \in H_{\Phi}^{n}[0,1]:\|x\|_{H \Phi} \leq r\right\}
$$

for some $r>0$ (see Section 2.f), then the boundary value problem (4.1) $L X+N x=0$ has at least one solution in $D_{L} \cap O^{2 n}[0,1] \cap B_{r}$.

Proof, - For the operator $N$ we state the following, with the proofs to be given later.

C) $N: H_{\Phi}^{n}[0,1] \rightarrow L_{1}[0,1]$ is continuous and bounded. 
D) $\left\|x_{1}\right\|^{2}+\left(N\left(J^{*} x_{1}+x_{0}\right), J^{*} x_{1}+x_{0}\right) \geqslant 0$ for $\left\|x_{1}\right\|^{2}+\left\|x_{0}\right\|^{2}=r^{2}>0$ where $x_{1} \in B_{1}$, $x_{0} \in S_{0}$. (We note: i) This computation is made in $L_{2}[0,1]$; ii) If $T=-T^{*}$, then $\left(J^{*} x_{1}+x_{0}, x_{1}\right)=0$. An assumption like $\left(J^{*} x_{1}+x_{0}, x_{1}\right)=0$ appears in Theorem 1 , Hypothesis a.)

Statements $(A)-(D)$ contain the key elements of the proof. We now just follow the proof of Theorem 3.1, making a few changes.

Statements $(A)$ and $(D)$ give the properties which carry us from (3.2) to (3.8), (3.9). This time

$$
P x=\sum_{1}^{k} z_{k} \int_{0}^{1} x(t) z_{k i}(t) d t \quad \text { where } z_{1}, \ldots, z_{l k}
$$

is an orthonormal basis for $S_{0}=N_{L} \subset L_{2}$. But also $z_{i} \in L_{\infty}, 1 \leq i \leq k$, so we can again, for convenience in later definition, regard $P$ as a projection on $L_{1}$.

As stated in $(A)$, the operators $H, J$ and $J^{*}$ are completely continuous integral operators, defined on $L_{2}[0,1]$-we shall not worry about their representations. If $N x \in C[0,1]$, then $H(I-P) N x \in C^{2 n}[0,1]$. And since $J$ and $J^{*}$ are integrals with $L^{\infty}$ kernels we can again define $K$ and $K^{*}$.

That $N: H_{\Phi}^{n} \rightarrow L_{1}$ is continuous and bounded is the same.

Again the mapping $T$ (see (3.12)) has

$$
T\left(z_{1}, x_{0}\right) \in A C[0,1] \quad \text { if } \quad\left(z_{1}, x_{0}\right) \in R_{1} \oplus R_{0}=L_{q}
$$

and again we regard this mapping on two levels:

$T: L_{\Phi} \rightarrow L_{\Phi}$ for fixed point theory, and

$T: L_{\Phi} \rightarrow L_{\Phi}$ for computing $\left\langle\left(z_{1}, x_{0}\right),(I+T)\left(z_{1}, x_{0}\right)\right\rangle$, since $L_{\Psi^{\prime}}^{*}=L_{\Phi}$.

By hypothesis $(a),\left(L_{\Psi^{\prime}}, L_{2}, L_{\Phi}\right)$ are in normal position (see Section 2.d.iv) so this pairing equals the $L_{2}$ inner product $\left(\left(z_{1}, x_{0}\right),(I+T)\left(z_{1}, x_{0}\right)\right)$ (The direct sum decomposition of $L_{2}=S_{1} \oplus S_{0}$ can be made a Hilbert space.)

Finally, the pairings that vanished by computation now vanish by orthogonality in $L_{2}$ and the proof is complete.

The following is an application of Theorem 4.1. Since $\Phi(u)=\exp \left(u^{9}\right)$ satisfies a $\Delta_{3}$-condition, its complementary $N$-function $\Psi(u)$ satisfies a $\Delta_{2}$-condition. Also, $\Psi(u)<u^{2}<\Phi(u)$. (See Sections 2.d.v and 2.d.iii.)

Theorem 4.2. - Let $f:[0,1] \times R \times R \times R \rightarrow R$ be continuous and assume: a) $\left.|f(t, x, y, z)| \leq a+b|x|+c|y|+d \exp |z|^{9} ; b\right)$ there exists an $r>0$ such that

$$
\int_{0}^{1}\left\{\left[x^{\prime \prime}(t)\right]^{2}+x(t) f\left(t, x(t), x^{\prime}(t), x^{\prime \prime}(t)\right)\right\} d t \geqslant 0 \quad \text { on } \partial B_{r}
$$

where $B_{r}=\left\{x \in H_{\Phi}^{2}[0,1]:\|x\|_{H \Phi} \leqq r\right\} . \quad\left(\Phi(u)=\exp \left(u^{9}\right)\right)$. 
Then the boundary value problem

$$
\begin{gathered}
x^{(i v)}+f\left(t, x, x^{\prime}, x^{\prime \prime}\right)=0 \\
x^{\prime}(0)=x^{\prime}(1)=x^{\prime \prime \prime}(0)=x^{\prime \prime \prime}(1)=0
\end{gathered}
$$

has at least one solution in $C^{4}[0,1] \cap B_{r}$.

A version of this theorem was announced in [10].

\section{BIBLIOGRAPHY}

[1] H. AMANN, Ein Existenz-und Eindeutigkeitssatz für die Hammersteinsche Gleichungen in Banachröumen, Math. Z., 111 (1969), pp. 175-190.

[2] J. W. BeBernes - K. SchMrT', Periodic boundary value problems for systems of second order differential equations, J. Diff. Eqs., 13 (1973), pp. 32-47.

[3] L. Cessari, Nonlinear Analysis, Bressanone, CIME Lecture Notes, 1972.

[4] L. Cesari - R. KANNAN, Functional analysis and nonlinear differential equations, Bull. Amer. Math. Soc, 79 (1973), pp. 1216-1219.

[5] T. Donaldoson - N. Trudinger, Orlicz-Bobolev spaces and embedding theorems, J. Funct. Anal., 8 (1971), pp. 52-75.

[6] P. HARTMaN, On boundary value problems for systems of ordinary, nonlinear, second order differential equations, Trans. Amer. Math. Soe., 96 (1960), pp. 493-509.

[7] P. Hartman, Ordinary Differential Equations, John Wiley, New York, 1964.

[8] J. K. Hale, Ordinary Differential Equations, Wiley-Interscience, New York, 1969.

[9] R. KANNAN - J. LoCKER, Nonlinear boundary value problems and operators TT* (to appear).

[10] R. KANNAN - J. SChUUR, Boundary value problems for even order nonlinear ordinary differential equations, Bull. Amer. Math. Soc., 82 (1976), pp. 80-82.

[11] H.-W. KNoBLoch, On the existence of periodic solutions for second order vector differential equations, 9 (1971), pp. 67-85.

[12] M. A. Krasnosmi'shin, Topological Methods in the Theory of Nonlinear Iniegral Equations, Pergamon-MacMillan, New York, 1964.

[13] M. A. Krasnosel'sicI - YA. B. RuTiCKI, Convex Functions and Onliez Spaces, P. Noordhooff Ltd., Groningen, Netherlands, 1961.

[14] J. Locker, The method of least squares for boundary value problems, Trans. Amer. Math. Soc., 154 (1971), pp. 57-68.

[15] J. MAWHIN, Nonlinear perturbations of Fredholm mappings in normed spaces and applieations to differential equations, Trabalho de Matematica No. 61, Univ. of Brasilia, 1974.

[16] M. Nagumo, Über die Differentialgleichung $y^{\prime \prime}=f\left(x, y, y^{\prime}\right)$, Proc. Phys.-Math. Soc. Japan (3) 19 (1937), pp. 861-866.

[17] K. Schmitr, Periodic solutions of systems of second-order differential equations, J. Diff. Eqs., 11 (1972), pp. 180-192. 